\title{
TENSOR PRODUCT OF COMMUTATIVE UNIONS OF GROUPS
}

\author{
JAMES A. ANDERSON AND NAOKI KIMURA
}

\begin{abstract}
In this paper a description is given of the tensor product of two arbitrary commutative unions of groups.
\end{abstract}

1. Structure theorem. The study of tensor products of commutative semigroups was initiated independently by Grillet [5] and Head [6]. In 1967, T. Head [8] determined the tensor product of a commutative group with a commutative union of groups, and posed the problem of determining the tensor product of semilattices, and more generally, the tensor product of commutative unions of groups. Anderson [1] treated closely related questions.

J. Delaney [4] determined the tensor product of semilattices when one of the semilattices is a chain. The solution for arbitrary semilattices (given below as Theorem 1.2) is due to N. Kimura [9], and the solution for complete lattices is forthcoming in N. Kimura and J. L. Williams [10].

In this paper, all groups and semigroups will be commutative, all semilattices will be lower semilattices with juxtaposition as the operation. Let $\phi(A, B)$ denote the set of finitely generated bifilters [2] of $A \times B$. The bifilter generated by $(\alpha, \beta) \in A \times B$ will be denoted by $[(\alpha, \beta)]$ or by $\alpha, \beta$ when used as a superscript or subscript. Let $\tau \uparrow S$ denote the restriction of $\tau$ to $S$. For general information on semigroups see [3], for bifilters see [2], and for colimits see [11].

Definition 1.1. Let $A$ and $B$ be semilattices and $C=A \times B$ be their direct product. A subset $F$ of $C$ is called a bifilter if the following conditions are satisfied:

(K0) $F$ is dual hereditary, i.e. if $(a, b) \in F, a \leqslant x, b \leqslant y$ then $(x, y) \in F$.

(K1) For every $b \in B$, if $(a, b),\left(a^{\prime}, b\right) \in F$ then $\left(a a^{\prime}, b\right) \in F$.

(K2) For every $a \in A$, if $(a, b),\left(a, b^{\prime}\right) \in F$ then $\left(a, b b^{\prime}\right) \in F$.

The following theorem is due to N. Kimura [9] and is given here without proof:

THEOREM 1.2. Let $A$ and $B$ be semilattices. Let $\mu: A \times B \rightarrow \phi(A, B)$ be the mapping defined by $\mu(\alpha, \beta)=[(\alpha, \beta)]$, then $\mu$ is $a$ bihomomorphism and $\phi(A, B)$ is the tensor product of $A$ and $B$.

Presented to the Society, January 17, 1974 under the title Tensor product of commutative semigroups which are unions of groups; received by the editors September 8, 1976.

AMS (MOS) subject classifications (1970). Primary 20M10.

(c) 1979 American Mathematical Society 0002-9939/79/0000-0051/\$02.75 
In the remainder of this paper $\phi(A, B)$ will be identified with $A \otimes B$.

Every union of groups can be completely characterized by its greatest semilattice quotient $A$, the maximal subgroup $G_{\alpha}$ for each $\alpha \in A$, and the system of homomorphisms $\phi_{\alpha^{\prime}}^{\alpha}: G_{\alpha} \rightarrow G_{\alpha^{\prime}}$ for $\alpha \geqslant \alpha^{\prime}$. Call $\left(G_{\alpha}, \phi_{\alpha^{\prime}}^{\alpha}, A\right)$ the corresponding triple of $G$, and $\left\{\phi_{\alpha^{\prime}}^{\alpha}\right\}$ the set of defining homomorphisms of $G$. Let $G_{\alpha}$ be the group associated with $\alpha$. Let $1_{\alpha}$ be the identity of $G_{\alpha}$, then $\phi_{\alpha^{\prime}}^{\alpha}\left(g_{\alpha}\right)=1_{\alpha^{\prime}} g_{\alpha}$. The semilattice of identities of the groups in $G$ will be identified with the greatest semilattice image of $G$.

In the remainder of this paper, $G$ and $H$ will be unions of groups. with corresponding triples $\left(G_{\alpha}, \phi_{\alpha^{\prime}}^{\alpha}, A\right)$ and $\left(H_{\beta}, \theta_{\beta^{\prime}}^{\beta}, B\right)$ resp.

Lemmas 1.3, 1.4, and 1.5 are due to Head [6], [7].

LEMMA 1.3. For semigroups $S$ and $T$, with greatest idempotent images $A$ and $B$ resp., $A \otimes B$ is the greatest idempotent image of $S \otimes T$.

LEMMA 1.4. If $S_{\alpha}$ and $T_{\beta}$ are maximal subgroups of semigroups $S$ and $T$, then $S_{\alpha} \otimes T_{\beta}$ is imbedded in $S \otimes T$ and is a maximal subgroup of $S \otimes T$.

LEMMA 1.5. The tensor product $G \otimes H$ is a union of groups.

LEMMA 1.6. $G_{\alpha} \otimes H_{\beta}$ is the group associated with $[(\alpha, \beta)]$ in $G \otimes H$.

Proof. From Lemma 1.4, $G_{\alpha} \otimes H_{\beta} \subseteq G \otimes H$. Hence by Lemma 1.5, $G_{\alpha} \otimes H_{\beta}$ is a maximal group in the union of groups $G \otimes H$.

From the commutivity of the diagram

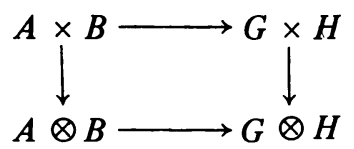

we have $G_{\alpha} \otimes H_{\beta}$ associated with $[(\alpha, \beta)]$ in $G \otimes H$.

Let $G$ and $H$ be unions of groups with corresponding triples $\left(G_{\alpha}, \phi_{\alpha^{\prime}}^{\alpha}, A\right)$ and $\left(H_{\beta}, \theta_{\beta^{\prime}}^{\beta}, B\right)$ resp. For $\Delta \in \phi(A, B)$ let $\Im_{\Delta}=\left\{G_{\alpha} \otimes H_{\beta}, \phi_{\alpha^{\prime}}^{\alpha} \otimes \theta_{\beta^{\prime}}^{\beta}(\alpha, \beta)\right.$, $\left.\left(\alpha^{\prime}, \beta^{\prime}\right) \in \Delta\right\}$. Let $T_{\Delta}$ be the colimit of $\mathfrak{S}_{\Delta}$ with colimiting cone $\left\{\gamma_{\Delta}^{(\alpha, \beta)}\right.$ : $\left.G_{\alpha} \otimes H_{\beta} \rightarrow T_{\Delta} \mid(\alpha, \beta) \in \Delta\right\}$. Choose $T_{\alpha, \beta}=G_{\alpha} \otimes H_{\beta}$ with colimiting cone $\left\{\phi_{\alpha}^{\alpha^{\prime}} \otimes \theta_{\beta}^{\beta^{\prime}}: G_{\alpha^{\prime}} \otimes H_{\beta^{\prime}} \rightarrow G_{\alpha} \otimes H_{\beta} \mid\left(\alpha^{\prime}, \beta^{\prime}\right) \in[(\alpha, \beta)]\right\}$. For $\Gamma, \Delta \in \phi(A, B)$, $\Gamma \subseteq \Delta \Rightarrow \mathfrak{S}_{\Gamma} \subseteq \mathfrak{S}_{\Delta}$. For each $(\alpha, \beta) \in \Gamma, \gamma_{\Delta}^{(\alpha, \beta)}$ is a homomorphism from $G_{\alpha} \otimes H_{\beta}$ to $T_{\Delta}$. Also by definition of colimiting cone, $\gamma_{\Delta}^{\left(\alpha^{\prime}, \beta^{\prime}\right)}\left(\phi_{\alpha^{\prime}}^{\alpha} \otimes \theta_{\beta^{\prime}}^{\beta}\right)=$ $\gamma_{\Delta}^{(\alpha, \beta)}$ for all $(\alpha, \beta),\left(\alpha^{\prime}, \beta^{\prime}\right) \in \Delta$ and hence for all $(\alpha, \beta),\left(\alpha^{\prime}, \beta^{\prime}\right) \in \Gamma$. Therefore by definition of colimit there exists $\sigma_{\Delta}^{\Gamma}: T_{\Gamma} \rightarrow T_{\Delta}$ such that

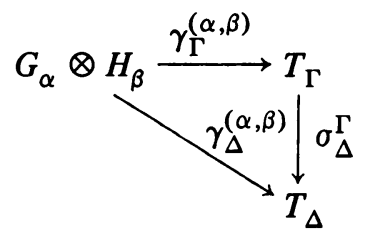


commutes for all $(\alpha, \beta) \in \Gamma$. Trivially $\sigma_{\Delta}^{\Gamma} \sigma_{\Gamma}^{\Lambda}=\sigma_{\Delta}^{\Lambda}$ hence we have the characterization of a union of groups.

LEMMA 1.7. $\left(T_{\Delta}, \sigma_{\Delta}^{\Gamma}, \phi(A, B)\right)$ is the corresponding triple for a union of groups, where $\Delta<\Gamma$ if $\Gamma \subseteq \Delta$.

Notice that $\sigma_{\alpha^{\prime} \beta^{\prime}}^{\alpha \beta}=\phi_{\alpha^{\prime}}^{\alpha} \otimes \theta_{\beta^{\prime}}^{\beta}$.

THEOREM 1.8. $T$ is the tensor product of $G$ and $H$.

2. Proofs. The proof of Theorem 1.8 is given through a series of lemmas.

Lemma 2.1. Define $\tau: G \times H \rightarrow T$ by $\tau\left(g_{\alpha} \otimes h_{\beta}\right)=g_{\alpha} \otimes h_{\beta} \in T_{\alpha, \beta}$ for $g_{\alpha} \in G_{\alpha}, h_{\beta} \in H_{\beta}$. The map $\tau$ is a bihomomorphism.

Proof. Let $\tau\left(g_{\alpha}, h_{\beta}\right)=g_{\alpha} \otimes h_{\beta} \in T_{\alpha, \beta}$ and $\tau\left(g_{\alpha}, h_{\beta^{\prime}}\right)=g_{\alpha} \otimes h_{\beta^{\prime}} \in T_{\alpha, \beta^{\prime}}$

Then

$$
\begin{aligned}
\tau\left(g_{\alpha}, h_{\beta}\right) \tau & \left(g_{\alpha}, h_{\beta^{\prime}}\right)=\sigma_{\alpha, \beta \beta^{\prime}}^{\alpha, \beta}\left(g_{\alpha} \otimes h_{\beta}\right) \sigma_{\alpha, \beta \beta^{\prime}}^{\alpha, \beta^{\prime}}\left(g_{\alpha} \otimes h_{\beta^{\prime}}\right) \\
& =\phi_{\alpha}^{\alpha} \otimes \theta_{\beta \beta^{\prime}}^{\beta}\left(g_{\alpha} \otimes h_{\beta}\right) \phi_{\alpha}^{\alpha} \otimes \theta_{\beta \beta^{\prime}}^{\beta^{\prime}}\left(g_{\alpha} \otimes h_{\beta^{\prime}}\right) \\
& =\left(g_{\alpha} \otimes \theta_{\beta \beta^{\prime}}^{\beta}\left(h_{\beta}\right)\right)\left(g_{\alpha} \otimes \theta_{\beta \beta^{\prime}}^{\beta^{\prime}}\left(h_{\beta^{\prime}}\right)\right) \\
& =g_{\alpha} \otimes \theta_{\beta \beta^{\prime}}^{\beta}\left(h_{\beta}\right) \theta_{\beta \beta^{\prime}}^{\beta^{\prime}}\left(h_{\beta^{\prime}}\right)=g_{\alpha} \otimes h_{\beta} h_{\beta^{\prime}}=\tau\left(g_{\alpha}, h_{\beta} h_{\beta^{\prime}}\right) .
\end{aligned}
$$

Similarly $\tau\left(g_{\alpha}, h_{\beta}\right) \tau\left(g_{\alpha^{\prime}}, h_{\beta}\right)=\tau\left(g_{\alpha} g_{\alpha^{\prime}}, h_{\beta}\right)$.

LEMMA 2.2. Let $G$ be a union of groups with corresponding triple $\left(G_{\alpha}, \phi_{\alpha^{\prime}}^{\alpha}, A\right)$ and $f: G \rightarrow S$ be a homomorphism, then $f(G)$ is a union of groups. If $\left(f\left(G_{\alpha}\right)\right.$, $\left.\bar{\phi}_{\alpha^{\prime}}^{\alpha}, f(A)\right)$ is the corresponding triple of $f(G)$ then

commutes.

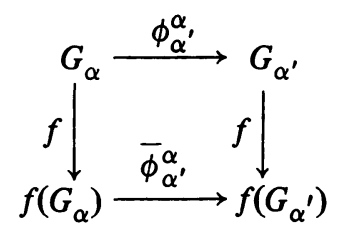

PROOF. $f \phi_{\alpha^{\prime}}^{\alpha}\left(g_{\alpha}\right)=f\left(1_{\alpha^{\prime}} g_{\alpha}\right)=f\left(1_{\alpha^{\prime}}\right) f\left(g_{\alpha}\right)=\bar{\phi}_{\alpha^{\prime}}^{\alpha} f\left(g_{\alpha}\right)$.

LEMma 2.3. Given a bihomomorphism $k: G \times H \rightarrow K$, there exists a unique homomorphism $f: T \rightarrow K$ such that $k=f \tau$.

Proof. Let $K$ be a semigroup and $k: G \times H \rightarrow K$ be a bihomomorphism. Let $f_{\alpha \beta}$ be defined on $G_{\alpha} \otimes H_{\beta}\left(=T_{\alpha, \beta}\right)$ so that

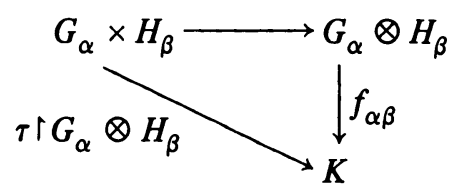

commutes. For $\Delta=\left[\left(\alpha_{1}, \beta_{1}\right) \ldots\left(\alpha_{n}, \beta_{n}\right)\right]$, let $K_{\Delta}$ be the maximal subgroup 
containing

$f_{\alpha(1) \beta(1)}\left(G_{\alpha(1)} \otimes H_{\beta(1)}\right) f_{\alpha(2) \beta(2)}\left(G_{\alpha(2)} \otimes H_{\beta(2)}\right) \cdots f_{\alpha(m) \beta(m)}\left(G_{\alpha(m)} \otimes H_{\beta(m)}\right)$. Let $\bar{K}=\cup K_{\Delta}$ and $\left(K_{\Delta}, \Psi_{\Delta}^{\Gamma}, \phi(A, B)\right)$ be the corresponding triple for $\bar{K}$. For $(\alpha, \beta) \in \Delta$, let $m_{\alpha \beta}: G_{\alpha} \otimes H_{\beta} \rightarrow K_{\Delta}$ be defined by $m_{\alpha \beta}=\Psi_{\Delta}^{\alpha \beta} f_{\alpha \beta}$. Using Lemmas 1.5, 1.6 and 2.2 one shows $\Psi_{\alpha^{\prime}, \beta^{\prime}}^{\alpha, \beta} f_{\alpha \beta}=f_{\alpha^{\prime} \beta^{\prime}}\left(\phi_{\alpha^{\prime}}^{\alpha} \otimes \theta_{\beta^{\prime}}^{\beta}\right)$. Hence

$$
\begin{aligned}
m_{\alpha \beta} & =\Psi_{\Delta}^{\alpha, \beta} f_{\alpha \beta}=\Psi_{\Delta}^{\alpha^{\prime}, \beta^{\prime}} \Psi_{\alpha^{\prime}, \beta^{\prime}}^{\alpha, \beta} f_{\alpha \beta} \\
& =\Psi_{\Delta}^{\alpha^{\prime}, \beta^{\prime}} f_{\alpha^{\prime} \beta^{\prime}}\left(\phi_{\alpha^{\prime}}^{\alpha} \otimes \theta_{\beta^{\prime}}^{\beta}\right)=m_{\alpha^{\prime} \beta^{\prime}}\left(\phi_{\alpha^{\prime}}^{\alpha} \otimes \theta_{\beta^{\prime}}^{\beta}\right) .
\end{aligned}
$$

Therefore by definition of colimit, there exists $f_{\Delta}: T_{\Delta} \rightarrow K_{\Delta}$ such that

$$
G_{\alpha} \otimes H_{\beta} \stackrel{\gamma_{\Delta}^{(\alpha, \beta)}}{m_{\alpha \beta}} T_{\downarrow_{\Delta}}^{f_{\Delta}}
$$

commutes for all $(\alpha, \beta) \in \Delta$. Define $f: T \rightarrow K$ such that $f$ agrees with $f_{\Delta}$ on $T_{\Delta}$.

By definition of $m_{\alpha \beta}$ we have for all $(\alpha, \beta) \in \Gamma \subseteq \Delta$

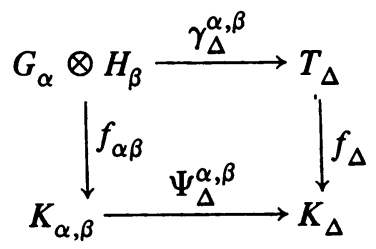

commutes. Hence in the diagram

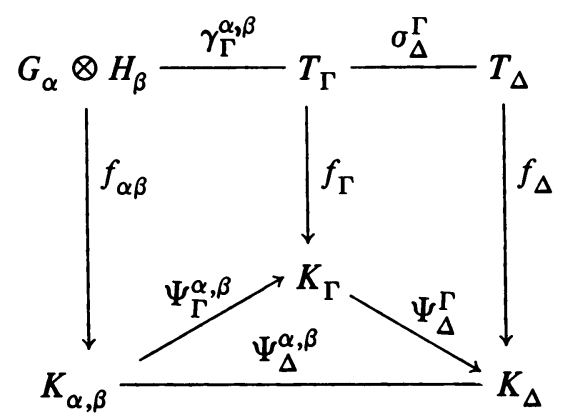

$f_{\Delta} \sigma_{\Delta}^{\Gamma} \gamma_{\Gamma}^{\alpha, \beta}=\Psi_{\Delta}^{\alpha, \beta} f_{\alpha \beta}=\Psi_{\Delta}^{\Gamma} \Psi_{\Gamma}^{\alpha, \beta} f_{\alpha \beta}=\Psi_{\Delta}^{\Gamma} f_{\Gamma} \gamma_{\Gamma}^{(\alpha, \beta)}$ for all $(\alpha, \beta) \in \Gamma$. Hence by definition of colimit $f_{\Delta} \sigma_{\Delta}^{\Gamma}=\Psi_{\Delta}^{+} f_{\Gamma}$. Hence

commutes.

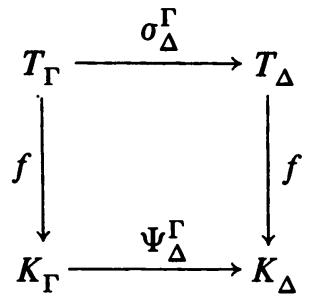


The function $f$ is a homomorphism, for let $u \in T_{\Gamma}, v \in T_{\Delta}, u v \in T_{\Lambda}$ then

$$
\begin{aligned}
f(u) f(v) & =\Psi_{\Lambda}^{\Gamma}\left(f(u) \Psi_{\Lambda}^{\Delta} f(v)\right)=f\left(\sigma_{\Lambda}^{\Gamma}(u)\right) f\left(\sigma_{\Lambda}^{\Delta}(v)\right) \\
& =f\left(\sigma_{\Lambda}^{\Gamma}(u) \sigma_{\Lambda}^{\Delta}(v)\right)=f(u v) .
\end{aligned}
$$

Trivially $f \tau=\beta$. Also $f$ is the unique homomorphism such that $f \tau=\beta$ since the image of $\tau$ generates $T$.

3. Remarks. This work also solves the structure of the tensor product of an arbitrary commutative semigroup with a union of groups. This follows since Head [7] has shown that if $S$ is an arbitrary commutative semigroup and $G$ a union of groups then

$$
(S \otimes G) \cong S \otimes(G \otimes Z) \cong(S \otimes Z) \otimes G
$$

and $S \otimes Z$ is a union of groups.

Since the tensor product of unions of groups in the category of commutative semigroups is a union of groups, it is the same in the category of unions of groups, hence this work also solves the structure of the tensor product of unions of groups in the category of commutative unions of groups.

\section{REFERENCES}

1. J. A. Anderson, Tensor product of a semigroup with a union of groups, Semigroup Forum 8 (1974), 65-68.

2. J. A. Anderson and N. Kimura, Tensor product of semilattices, Semigroup Forum 11 (1978), 83-88.

3. A. H. Clifford and G. B. Preston, The algebraic theory of semigroups, Math. Surveys, no. 7, Vol. 1, Amer. Math. Soc., Providence, R.I., 1961.

4. J. E. Delaney, The tensor product of semilattices, Portugal. Math. 31 (1972), 193-202.

5. P. A. Grillet, The tensor product of commutative semigroups, Trans. Amer. Math. Soc. 138 (1969), 267-280.

6. T. J. Head, Homomorphisms of commutative semigroups as tensor maps, J. Natur. Sci. and Math. 7 (1967), 38-49.

7. (1969), fasc. $1-4$.

8. $155-159$.

9. N. Kimura, Tensor product of semilattices, Notices Amer. Math. Soc. 17 (1970), 554.

10. N. Kimura and J. L. Williams, Tensor product of complete semilattices (to appear).

11. S. Mac Lane, Categories for the working mathematician, Springer-Verlag, New York, 1971.

Department of Mathematics, Northern Arizona University, Flagstaff, Arizona 86011

Department of Mathematics, University of Arkansas, Fayetteville, Arkansas 72701 